http://dx.doi.org/10.35381/e.k.v3i6.814

\title{
Rutas y protocolos como sensores de violencia entre pares
}

\section{Routes and protocols as sensors of violence between peers}

\author{
María Esther Regalado-Martínez \\ maria.regalado@psg.ucacue.edu.ec \\ Universidad Católica de Cuenca, Azogues \\ Ecuador \\ https://orcid.org/0000-0003-3714-8933 \\ Darwin Gabriel García-Herrera \\ dggarciah@ucacue.edu.ec \\ Universidad Católica de Cuenca, Azogues \\ Ecuador \\ https://orcid.org/0000-0001-6813-8100 \\ Sergio Constantino Ochoa-Encalada \\ scochoae@ucacue.edu.ec \\ Universidad Católica de Cuenca, Cuenca \\ Ecuador \\ https://orcid.org/0000-0003-3067-3719 \\ Juan Carlos Erazo-Álvarez \\ jcerazo@ucacue.edu.ec \\ Universidad Católica de Cuenca, Cuenca \\ Ecuador \\ https://orcid.org/0000-0001-6480-2270
}

Recepción: 09 abril 2020

Revisado: 15 de mayo 2020

Aprobación: 12 junio 2020

Publicación: 1 de julio 2020 


\title{
RESUMEN
}

La investigación tuvo por objetivo comprender la percepción que tienen sobre las rutas y los protocolos de actuación como sensores de violencia entre pares, los profesionales que hacen vida en el DECE de la Unidad Educativa La Providencia de la ciudad de Azogues de Ecuador. El estudio se desarrolló desde un enfoque interpretativo-cualitativo, con base en el método etnográfico. A partir de las categorias emergentes se puede expresar que por parte de la comunidad educativa existe fallta de conocimiento sobre las rutas y protocolos de violencia entre; asimismo, y desde lo manifestado por el personal del Departamenteo de Consejería Estudiantil, es de vital importancia otorgarle un tinte humanistico a la formulacion de estas rutas y protocolos como manuales que orientan la práctica para la atención de la violencia entre pares, siendo indispensable para ello la participación de psicólogos en estos campos de acción.

Descriptores: Asesoramiento; violencia; relaciones interpersonales; relación profesoralumno. (Palabras tomadas del Tesauro UNESCO).

\begin{abstract}
The objective of the research was to understand the perception that the professionals who make life in the DECE of the La Providencia Educational Unit of the city of Azogues in Ecuador have about the routes and the protocols of action as sensors of violence between peers. The study was developed from an interpretive-qualitative approach, based on the ethnographic method. From the emerging categories it can be expressed that on the part of the educational community there is a lack of knowledge about the routes and protocols of violence between; Likewise, and from what was stated by the staff of the Student Counseling Department, it is of vital importance to give a humanistic tinge to the formulation of these routes and protocols as manuals that guide the practice for the attention of peer violence, being essential for this the participation of psychologists in these fields of action.
\end{abstract}

Descriptors: Counselling; violence; interpersonal relations; student teacher relationship. (Wordstakenfromthe UNESCO Thesaurus). 
María Esther Regalado-Martínez; Darwin Gabriel García-Herrera; Sergio Constantino Ochoa-Encalada; Juan Carlos Erazo-Álvarez

\section{INTRODUCCIÓN}

El ser humano es un individuo biopsicosocial y trascendente, que resulta de la combinación de factores determinantes tanto de la naturaleza genética-orgánica como de las caracteristicas personales y ambientales, que actuan en el desarrollo. Es de saber que producto de estas interacciones se producen respuestas adaptativas, pero, tambien desadaptativas, siendo una de ellas la violencia que se constituye en un problema en la relación interpersonal, convirtiendose en una situacion negativa dentro de la tarea global del individuo que consiste en adquirir una identidad positiva (Fuertes-Grábalos, et al., 2017). Pues, como refieren (Papalia,et al., 2010), lo largo del ciclo vital, el ser humano debe cumplir con una serie de tareas como eventos normativos y que en su superación va formando su personalidad de manera funcional y adaptada a su entorno; sin embargo, existen una serie de acontecimientos como los eventos no normativos que podrían constituir un obstáculo en dicho proceso y que incluso influiría de manera significativa en la visión de sí mismo y del contexto.

Es de saber que etimológicamente la palabra violencia viene del latín violentus, que significa el que actúa con mucha fuerza. Por consiguiente y considerando a la (MartínezPacheco, 2016), la violencia refiere al uso intencional de la fuerza física, amenazas contra uno mismo, otra persona, un grupo o una comunidad que tiene como consecuencia, o es muy probable que tenga como consecuencia, un traumatismo, daños psicológicos, problemas de desarrollo o la muerte.

Para el psicoanálisis las manifestaciones violentas tienen su origen en las pulsiones instintivas del ello. En este sentido, Winnicott psicoanalista británico postula que el verdadero problema radica en cómo controlar, canalizar o sublimar la agresión (ChagasDorrey, 2012). Pues el propio Winnicott plantea que la cuestion no es expresar la agresividad, sino su temprana represión en base a la ansiedad moral y ante la realidad que se hace presente; pues como impulso primario agresivo producto de la energía libidinal del ser humano, tiene la necesidad de ser expresada y no de ser contenida para evitar la formación de la llamada agresión reactiva. 
María Esther Regalado-Martínez; Darwin Gabriel García-Herrera; Sergio Constantino Ochoa-Encalada; Juan Carlos Erazo-Álvarez

Cabe decir, que el Ministerio de Educación (MINEDUC, 2017) define la violencia como un suceso que se suscita en el seno de un conflicto y en la trama de las relaciones inequitativas de poder y sometimiento que puede ocurrir en diversos escenarios (p.19); por consiguiente, los problemas de violencia son inherentes a la realidad de las relaciones interpersonales. Es por ello que (Barquero-Brenes, 2014), indica que la violencia es un fenómeno que ejerce poder bajo una figura estricta y abusiva que pretende ocasionar daño de manera deliberada; por lo tanto, amerita ser atendida de manera oportuna y eficaz.

De igual forma (Caballero-Grande, 2010), expresa que la presencia de manifestaciones de violencia representa un conflicto que afecta la convivencia, un aspecto importante en el desarrollo de las relaciones humanas que resulta de la interacción entre los individuos que se constituye por lazos de consanguinidad, afinidad o mandatos legales. En este mismo sentido, el propio (Barquero-Brenes, 2014) dice que la confianza y el respeto son la base de la convivencia para la construcción de una cultura de paz; sin embargo, no podemos obviar que vivimos en una sociedad donde los conflictos, a veces, no suelen tener una solución exitosa y es ahí donde se da lugar a manifestaciones violentas que representan un riesgo evidente.

Es asi que la violencia teniendo un origen en la conducta imitativa, puede constituirse en un grave problema en las diversas esferas del desarrollo; no obstante, hay que tener presente que el desarrollo del ser humano esta encadenado a procesos formativos en el marco del aprendizaje humano. En este ambito, cada uno de los individuos puede superar los acontecimientos que se le presentan en su vida, sin embargo, si hay conflictos que no permiten su desarrollo adecuado, los sujetos posiblemente manifesten conductas violentas, o, se convierten en víctimas.

Es por ello que La Declaración Universal de Derechos Humanos plantea que todos los pueblos y nacionalidades promuevan valores de enseñanza, educación y respeto a las libertades individuales y colectivas, valiéndose de medidas progresivas de carácter nacional e internacional y su aplicación universal y efectiva. En otras palabras, busca 
precautelar la integridad de cada individuo, considerando el cumplimiento de los artículos (del 1 al 30) proclamados por la asamblea general en materia de Derechos Humanos (DDHH). Puediendose resaltar de alli, especificamente el artículo 5, el cual hace especial énfasis en las diversas formas de violencia que podrían manifestarse como formas de torturas, tratos crueles, inhumanos o degradantes (Organización de las Naciones Unidas, 2015).

Al respecto, en el contexto ecuatoriano, la Comisión de Transición hacia el Consejo de las mujeres y la igualdad de género, clasifica la violencia como: física, psicológica, sexual, por omisión y virtual. No obstante, en el ambito educativo las formas de violencia desde un enfoque interpersonal se han conceptuado como: intrafamiliar, institucional, entre pares, sexual y de estudiantes contra adultos

Ahora bien, lo planteado al contextualizarlo al ambito educativo implica hacer algunas precisiones de hechos sucedidos. En este sentido, (Hernandez, 2018a) evidencia con su investigación, que el 36,3\% de niñas y niños fueron víctimas de violencia por parte de sus compañeros dentro del entorno educativo; además, que el 75,7 \% de estos actos de violencia psicológica o física ocurrieron en el aula de clases; por lo que el psicólogo es una figura clave en la intervención preventiva y de promoción escolar para la mejora del clima en el aula, valiendose del diálogo asertivo y la capacidad para resolver problemas. Tambien, es significativo resaltar lo que dicen (Pineda-Almaraz, et al., 2015) sobre la percepción de Bullying como expresión de violencia entre los estudiantes, profesores de primaria y secundaria; en este sentido, la percepción entre los grupos es muy contradictoria, esto debido a que los estudiantes lo identifican como una situación que se presenta frecuentemente y es grave; en cambio los profesores perciben a este fenómeno como algo que se muestra con baja intensidad en los centros educativos y de manera aislada, concluyendo que estas diferencias presentadas pueden distorsionar una convivencia adecuada dentro del contexto escolar.

En el marco de lo expuesto y adentrando al contexto ecuatoriano, es importante referenciar que en el año 2015 el propio MINEDUC, UNICEF y Visión Mundial realizaron 
una investigación en diferentes instituciones educativas del Ecuador acerca de la violencia entre pares, los resultados evidencian que uno de cada cinco estudiantes afirma haber sido víctimas de violencia escolar (Vera-Mora \& León-Acurio, 2017).

Situaciones como la planteada que demuestran el creciente auje de la violencia en el ambito educativo, son las que han justificado la implementacion de una real politica de intervención en esta materia. En este sentido, es importante señalar que la Constitución de la República del Ecuador expresa que "La educación se centrará en el ser humano y garantizará su desarrollo holístico, en el marco del respeto a los derechos humanos, al medio ambiente sustentable y a la democracia; será participativa, obligatoria, intercultural, democrática, incluyente y diversa (...)" (Registro Oficial 449 de 20-oct-2008). De alli que la educación se considere un proceso de interacción social, y como tal conlleva un conjunto de parametros que pueden facilitarla o dificultarla. Por ese motivo, el (MINEDUC, 2017) ha planteado los "Protocolos de actuación frente a la violencia detectadas o cometidas en el Sistema Educativo" que se han de asumir como rutas a seguir y sensores de dicho fenómeno.

Este instrumento de protocolos de actuación, que fundamentado de manera teórica, legal y metodologicamente, contiene las directrices para el abordaje de este fenómeno; además, de un conjunto de estrategias y pasos a seguir para intervenir de manera oportuna las situaciones de violencia en el marco de la resolución de conflictos, tanto a nivel preventivo como de carácter reactivo; por tanto, se constituyen en objetos de estudio a proposito de comprender y mejorar sus particularidades.

En este contexto y según el acuerdo ministerial 00046-A (MINEDUC, 2016) se ha estipulado que el Departamento de Consejería Estudiantil (DECE de aquí en adelante). es quien debe cumplir con las funciones de asesoría y acompañamiento psicopedagógico y emocional; protección y asistencia oportuna en estudiantes a los que se ha vulnerado sus derechos y seguimiento de los casos según el "Modelo de Atención Integral de los Departamentos de Consejería Estudiantil" Siendo indispensable aplicar pruebas para conocer las motivaciones de los estudiantes en cuanto al proyecto de vida como meta 
María Esther Regalado-Martínez; Darwin Gabriel García-Herrera; Sergio Constantino Ochoa-Encalada; Juan Carlos Erazo-Álvarez

para trabajar en un crecimiento personal integral (Romero \& Villasmil, 2017). De la misma manera, expresa que este debe regirse por protocolos en materia de promoción y prevención de problemáticas sociales; por lo tanto, debe hace uso de ellos cuando lo amerite.

Sin embargo, la visión del personal se ha visto dividida, pues dentro del ámbito legal se ha llegado a un consenso sobre la implementación de estos procedimientos, pero en su aplicación educativa se han presentado innumerables obstáculos para un abordaje idóneo y desde un enfoque humanístico, que busca restablecer y garantizar los derechos de los educandos, siendo factible para tal fin, el acompañamiento familiar (Hernández, 2018b). Por consiguiente, es fundamental visualizar la vinculación activa y cooperativa entre docentes, orientadores, autoridades y padres de familia en la intervención, para asi conocer actitudes, creencias y percepciones de los miembros de la comunidad educativa (Roque-Díaz, 2018), acerca de la concepción y aplicación de procesos y protocolos de atención de violencia entre pares en la convivencia (Sáez-de-Heredia, 1998).

Haciendo algunas consideraciones teoricas sobre este tema en el contexto lationamericano, se tiene que (Duarte-Duarte, 2003), expresa que desde la percepción de psicólogos y orientadores en cuanto al fortalecimiento de una adecuada aplicación de protocolos de intervención, existen ciertos aspectos que conforman la estructura de estos sistemas obstaculizan y afectan la implementación de dichos procedimientos a nivel organizativo, afectando así el logro de metas en el abordaje y solución de este fenómeno. Asimismo, (Alfaro-Molina, et al., 2010) con su estudio realizado en Costa Rica, con el objetivo de conocer el manejo y dominio en protocolos de intervención en violencia escolar, determinaron que la población no poseen un conocimiento adecuado sobre cómo detectar e intervenir en casos de violencia escolar; puesto que, tan solo un $16 \%$ de los que participaron en dicho estudio han recibido algún tipo de capacitación sobre este fenómeno.

Lo planteado se compagina con lo expresado por (Sánchez-Carranza \& Sandoval-Forero, 2016), en el sentido de que ellos sostienen que los orientadores realizan funciones más 
apegadas a trámites administrativos y al cumplimiento de normas y reglamentos escolares, que a la función de acompañamiento y asesoría; de igual manera, con lo dicho por (Mata-Diz \& Penido-Martins, 2017) respecto a que existen muchas diferencias entre los mismos profesionales al momento de conceptualizar este fenómeno; por lo tanto, al no presentar una misma percepción hace que los abordajes de los casos de violencia no sean eficaces y oportunos.

En lo que respecta al ambito ecuatoriano, es importante considerar a (Vera-Mora \& LeónAcurio, 2017) pues evidencian que las intervenciones oportunas del DECE reducen los impactos negativos producto de la violencia (miedo, apatía, bajo rendimiento académico, depresión, entre otra; por consiguiente, se deben socializar los diversos protocolos de violencia frente a casos existentes para un abordaje oportuno. Sin embargo, es de saber que (Neut, 2017) con sus hallazgos investigativos, enuncian que el personal de educación desconoce aspectos relevantes acerca de las rutas y protocolos para la detección e intervención de casos de violencia entre pares.

En virtud de lo estipulado, es importante saber que la Unidad Educativa Particular La Providencia de la provincia de Azogues conformó un DECE compuesto por las áreas de psicología educativa, clínica y trabajo social, con el propósito de atender de forma oportuna las demandas concernientes a los problemas de violencia presentes en el sistema educativo basandose en los rutas y protocolos de actuación establecidos por el ministerio. A pesar de ello, los profesionales de las áreas mencionadas, manifiestan inquietudes sobre ciertas ordenaciones que obedecen a los procedimientos de rigor, pues se evidencia que se han constituido en una traba para la resolución de conflictos.

En este sentido y considerando que los profesionales que hacen vida en esta institución estan inmersos en en esta práctica actuacional para la resolucion de los problemas de violencia es fundamental preguntarse: ¿Cómo son percibidas las rutas y los protocolos de actuación como sensores de violencia entre pares en la Unidad Educativa La Providencia de la ciudad de Azogues de Ecuador? De allí que la investigación se enmarca en comprender la percepción que tienen sobre las rutas y los protocolos de actuación 
María Esther Regalado-Martínez; Darwin Gabriel García-Herrera; Sergio Constantino Ochoa-Encalada; Juan Carlos Erazo-Álvarez

como sensores de violencia entre pares, los profesionales que hacen vida en el DECE de la Unidad Educativa La Providencia de la ciudad de Azogues de Ecuador.

\section{MÉTODO}

Esta investigación se desarrolló desde un enfoque interpretativo-cualitativo con base en el método etnográfico. En este sentido, se buscó comprender las rutas y los protocolos de actuación como sensores de violencia entre pares, por cuanto es un fenómeno que viven en la práctica cotidianidad los profesionales participantes del DECE de la Unidad Educativa La Providencia

Lo anterior define la investigacion con un diseño cacterizado por las cualidades de emergente y flexible, dado que asume como premisa para la construcción de conocimientos, la comprensión de acciones, eventos, acontecimientos, creencias, valores, significados, experiencias desde la perspectiva de los sujetos de investigación. Entonces, a partir de la vivencia de los sujetos de investigación, se generó un proceso observacional, para la construccion de perspectivas en torno al fenomeno de estudio desde la intersubjetividad como vía para acceder al sentido o significado como conocimiento que se construye en la vida cotidiana considerando al otro y en interacción con lo otro (Hernández-Romero \& Galindo-Sosa, 2007).

Para ello se constituyó un grupo de 5 personas o sujetos de investigación mediante la técnica de muestreo intencional considerando el criterio teórico de ser profesional participante del DECE de la Unidad Educativa La Providencia de la ciudad de Azogues y aplicar los protocolos de actuación frente a situaciones de violencia detectadas 0 cometidas en el sistema educativo.

La técnica empleada fue el grupo focal, el cual consiste en una entrevista que se realiza de manera grupal y donde los participantes conversan a profundidad en torno a uno 0 varios temas en un ambiente relajado e informal y con el objetivo de generar y analizar la interacción ente ellos y cómo se construyen grupalmente significados en relación al fenómeno abordado (Aldana-Zavala, 2019). Asimismo, con el grupo focal se busca que 
María Esther Regalado-Martínez; Darwin Gabriel García-Herrera; Sergio Constantino Ochoa-Encalada; Juan Carlos Erazo-Álvarez

los participantes expresarn sus opiniones sobre diferentes aspectos de interés en un ambiente abierto para el libre intercambio de ideas. Ademas, como expresan (GrossMartínez \& Stiller-González, 2015), de proveerles espacios de participación a las personas involucradas en los respectivos programas.

Para la recopilación de información se empleó un protocolo de entrevista con seis preguntas, previamente validado por psicologos expertos y se registró un consentimiento informado en el cual se especifica que todo el grupo focal fuese grabado bajo autorización para un mejor manejo de la información. Con respecto al procesamiento y analisis de la data recabada, es de saber que esta se dio mediante la construcción de unidades de analisis desde donde se develaron las categorias emergentes sobre el fenomeno y que fueron objeto de interpretacion. 
EPISTEME KOINONIA

Revista Electrónica de Ciencias de la Educación, Humanidades, Artes y Bellas Artes

Año III. Vol III. N ${ }^{\circ}$ 6. Julio - Diciembre 2020

Hecho el depósito de Ley: FA2018000022

ISSN: 2665-0282

FUNDACIÓN KOINONIA (F.K).

Santa Ana de Coro, Venezuela

María Esther Regalado-Martínez; Darwin Gabriel García-Herrera; Sergio Constantino Ochoa-Encalada; Juan Carlos Erazo-Álvarez

\section{RESULTADOS}

Se presentan los resultados en razón del tratatamiento interpretativo de la data recopilada:

\section{Tabla 1.}

Percepciones del grupo focal.

\begin{tabular}{|c|c|c|}
\hline $\begin{array}{l}\text { UNIDAD DE } \\
\text { ANÁLISIS }\end{array}$ & CATEGORÍA & SEGMENTO \\
\hline $\begin{array}{c}\text { Percepción } \\
\text { de } \\
\text { integrantes } \\
\text { del DECE }\end{array}$ & $\begin{array}{l}\text { Percepción } \\
\text { ante Rutas y } \\
\text { Protocolos. }\end{array}$ & $\begin{array}{l}\text { Existe negligencia por falta de socialización o desconocimiento de } \\
\text { - } \quad \text { El enfoque debe ser humanista y no imponer al estudiante. } \\
\text { - } \quad \text { La percepción del profesional del DECE es de mucha importancia } \\
\text { debido a que de ello depende la resolución. } \\
\text { - Las Rutas y Protocolos es una función netamente burocrática, se } \\
\text { pierde la funcionalidad del DECE. } \\
\text { - Este proceso limita a los profesionales realizar un trabajo eficaz y } \\
\text { - Entorpece los resultados. } \\
\text { - Existen varias percepciones de los integrantes del DECE. }\end{array}$ \\
\hline $\begin{array}{c}\text { Rutas y } \\
\text { protocolos }\end{array}$ & $\begin{array}{l}\text { Aporte para } \\
\text { nuevas } \\
\text { ediciones }\end{array}$ & $\begin{array}{l}\text { - La principal dificultad es que los maestros no tienen claro el } \\
\text { - Sroceso de aplicación de las rutas y protocolos. } \\
\text { - } \quad \text { Exceso de procesos burocráticos apartando a los profesionales de } \\
\text { su labor psicológica. } \\
\text { - Importancia de la participación de los psicólogos en la elaboración } \\
\text { debido a que es un tema de construcción psicosocial. } \\
\text { - Elaboración de profesionales de distinta índole, psicólogo, } \\
\text { abogado, miembros de la comunidad educativa. (Directivos. } \\
\text { Padres de familia y docentes). }\end{array}$ \\
\hline $\begin{array}{l}\text { Violencia } \\
\text { entre pares }\end{array}$ & Significación & $\begin{array}{l}\text { - La agresión puede ser física, psicológica y verbal. } \\
\text { - } \quad \text { un violencia entre pares es toda acción con la intención de causar } \\
\text { Intervienen varias persona. } \\
\text { espectadores quienes se involucran en el acto de violencia. }\end{array}$ \\
\hline
\end{tabular}

Fuente: Elaboración propia (2020). 
María Esther Regalado-Martínez; Darwin Gabriel García-Herrera; Sergio Constantino Ochoa-Encalada; Juan Carlos Erazo-Álvarez

A continuación, se presentan las categorias emergentes y su contrastación con referentes teoricos conceptuales que se han considerado previamente para una mejor comprension del fenomeno en estudio.

\section{Percepción de los psicólogos: Influencia en el proceso de resolución}

Para el personal del DECE de la Unidad Educativa Particular La Providencia, las rutas y protocolos de violencia entre pares debe ser abordada desde una visión mas humanista para el bienestar de la comunidad educativa, mas no deben ser visto como una imposición; por lo tanto, es de vital importancia que las autoridades a cargo los socialicen y los pongan a disposición del personal en cuestión.

Lo anterior se complementa con lo expuesto por (Alfaro Molina et al., 2010), (Vera-Mora \& León-Acurio, 2017), en relación a que existe un desconocimiento y desacuerdo con el manejo y aplicación de los protocolos a seguir en el caso de violencia entre pares; además, con lo planteado por (Vera-Mora \& León-Acurio, 2017) sobre el aspecto humanistico como factor importante en la implementación de estos procesos, para deslastrarse del carácter de obligatoriedad.

\section{Percepción ante Rutas y Protocolos}

La categoría percepción ante rutas y protocolos, evidencia que estos se han constituido en simples trámites burocráticos cuyo objetivo es obedecer disposiciones institucionales, que en muchos de los casos, entorpecen los procesos y como consecuencia no existe una resolución satisfactoria; esto se compagina con lo expresado por (Hernández, 2018a) en el sentido de que la práctica del psicólogo deberia ser más humanizada y no solo centrarse en los meros tramites administrativos. Ademas, con lo enunciado por (Mata-Diz \& Penido-Martins, 2017), pues manifiestan que existen diversas perspectivas en el momento de abordar los temas que involucran esta categoría. 
María Esther Regalado-Martínez; Darwin Gabriel García-Herrera; Sergio Constantino Ochoa-Encalada; Juan Carlos Erazo-Álvarez

\section{Rutas y Protocolos: Dificultad de aplicación}

Con base en la data analizada, las principales dificultades que surgen en la aplicación de las Rutas y Protocolos de violencia entre pares son: el desconocimiento, el exceso de trámites administrativos y burocráticos que han apartado a los funcionarios del DECE de su labor sustancial con respecto al bienestar de los estudiantes. Esto se puede consustanciar: por una parte, con lo expuesto por (Alfaro Molina et al., 2010), (Mata-Diz \& Penido-Martins, 2017), (Vera-Mora \& León-Acurio, 2017) exponen que el personal del DECE e incluso los docentes desconocen el verdadero propósito de estos manuales y los han considerado como meros tramites administrativos. Por otra parte, con lo que expresa (Pineda-Almaraz, et al., 2015) en relacion a que los psicólogos u orientadores figuran como personal destinados a cumplir funciones burocráticas alejandose de su verdadero objetivo.

\section{Aporte para nuevas ediciones}

Desde la visión de los participantes, la intervención del personal del DECE en la formulación de nuevas ediciones de rutas y protocolos de violencia entre pares, debe ser tomada en cuenta como uno de sus principales ejes; ya que la violencia es una construcción psicosocial que recae enteramente en el campo de la psicología. Adicional a esto, se necesita de la asesoría del personal jurídico y otros miembros de la comunidad educativa como sujetos que tambien viven elfenómeno. El papel primordial del DECE con base en la psicología va más allá de ejercer una función administrativa; no obstante, debe ejercer una labor humanizada y humanizadora tomando en cuenta las particularidades de cada estudiante y asi lograr su desarrollo integral. 
María Esther Regalado-Martínez; Darwin Gabriel García-Herrera; Sergio Constantino Ochoa-Encalada; Juan Carlos Erazo-Álvarez

\section{Violencia entre pares: Significación}

Los participantes significan la violencia entre pares como prácticas de carácter reiterativo destinado a ocasionar daño de manera intencional pudiendo ser físico, psicológico y verbal. Además, ponen en manifiesto que existen tres posiciones frente a este fenómeno: víctima, agresor y observadores. Esta interpretacion se puede complementar con lo expresado por (Vera-Mora \& León-Acurio, 2017) pues ellos manifiestan que la violencia en el ámbito educativo puede ser de distintos tintes, más allá de un acto verbal y que provoca daño en la persona que está expuesta. 
María Esther Regalado-Martínez; Darwin Gabriel García-Herrera; Sergio Constantino Ochoa-Encalada; Juan Carlos Erazo-Álvarez

\section{PROPUESTA}

Finalmente, luego de comprender la percepcion de los profesionales que aplican las rutas y protocolos de violencia y de realizar una exahustiva revision bibliografica, se presenta la siguiente propuesta denominado PIC (Planificación - Implementación - Capacitación), para mejorar la estructuración de futuras versiones de Rutas y Protocolos, considerando la labor de los profesionales que hacen vida en los DECE, pues es menester de esta área trabajar la esfera psicológica (diagnóstico, tratamiento/intervencion, evaluación y seguimiento) de problemáticas en el ámbito educativo preocupandose de la persona mas allá de las aulas, y a fin de optimizar recursos y tiempo ante este problema.Dicha propuesta se detallada a continuación. 
EPISTEME KOINONIA

Revista Electrónica de Ciencias de la Educación, Humanidades, Artes y Bellas Artes

Año III. Vol III. N ${ }^{\circ}$ 6. Julio - Diciembre 2020

Hecho el depósito de Ley: FA2018000022

ISSN: 2665-0282

FUNDACIÓN KOINONIA (F.K).

Santa Ana de Coro, Venezuela

María Esther Regalado-Martínez; Darwin Gabriel García-Herrera; Sergio Constantino Ochoa-Encalada; Juan Carlos Erazo-Álvarez

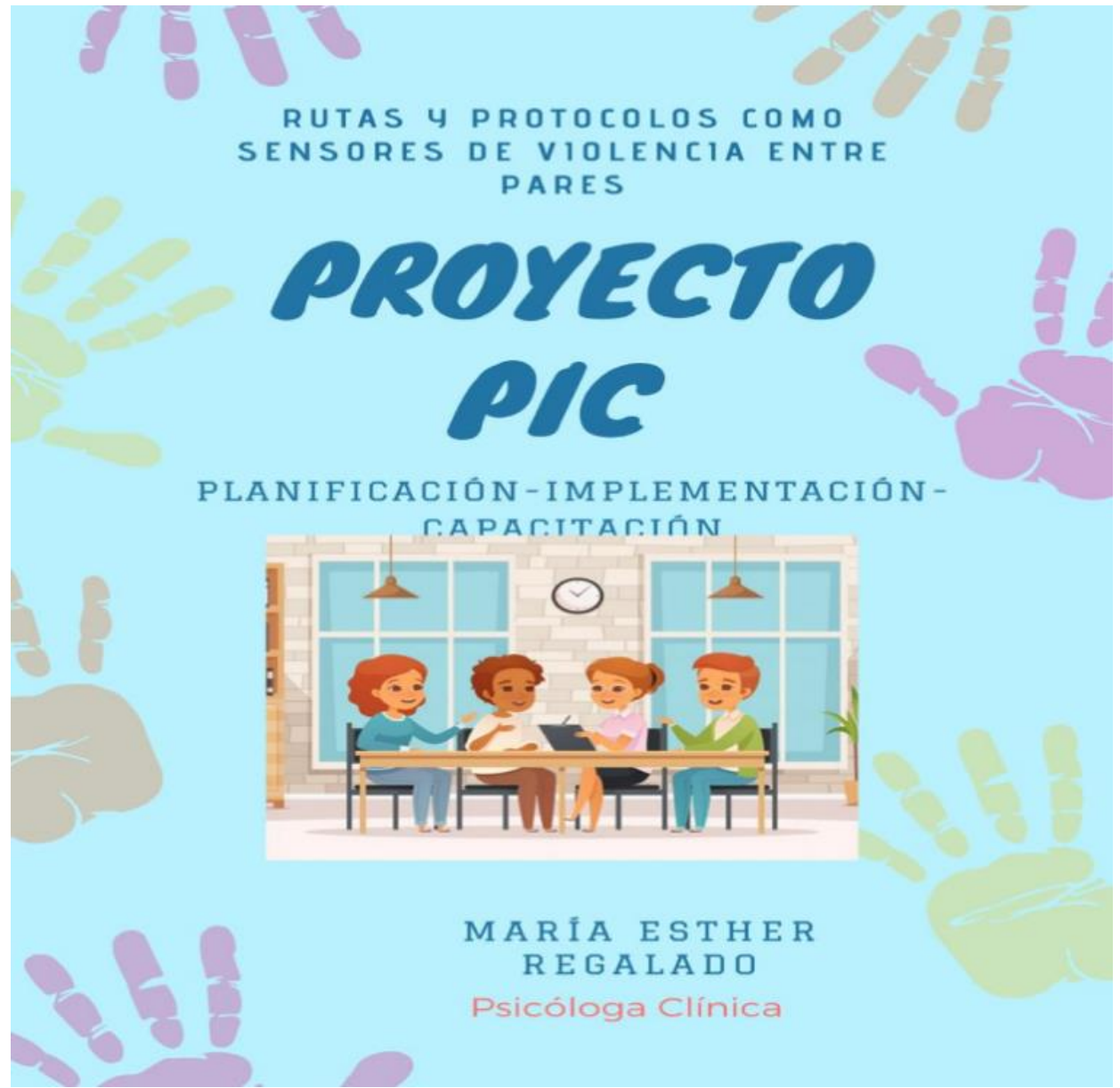

Figura 1. Proyecto PIC. Fuente: Elaboración propia (2020). 
Planificación: La planificación es un punto muy importante para el desarrollo de un proyecto, el mismo que irá dirigido a los profesionales de las comunidades educativas y más aún si se trata de un protocolo a seguir para la resolución de violencia entre pares. Es por ello que posterior a este estudio se ve la necesidad de realizar algunas modificaciones en estas rutas y protocolos de violencia entre pares como:

1. La intervención de la elaboración o mejoras de las rutas y protocolos debería ser propuestas por un equipo conformado por un miembro de la comunidad educativa, un psicólogo clínico que tenga experiencia en el DECE, un asesor legal, una autoridad educativa y un profesional que labore en el Ministerio de Educación.

2. Se deberá clasificar este protocolo haciendo distinción por edades debido a que los niños menores de 12 años son inimputables y con los mismos no se pueden ejecutar a plenitud las rutas, por lo que, se debería realizar otras rutas y protocolos para estos rangos de edad.

3. Buscar estrategias de abordaje y recolección de la información alejada de los excesos de trámites burocráticos que dejan de lado la búsqueda del bienestar estudiantil como principio teleológico.

Implementación: La implementación de estrategias de capacitación y mejoras en los profesionales en relación a las rutas y protocolos de violencia entre pares es fundamental, pues estos dependen del conocimiento y preparación para un correcto abordaje y seguimiento de los casos que a diario se presentan, siendo indispensable que conozcan sobre todo los pasos a seguir dentro de estos protocolos, pues los mismos facilitan el trabajo dentro de las comunidades educativas. 
María Esther Regalado-Martínez; Darwin Gabriel García-Herrera; Sergio Constantino Ochoa-Encalada; Juan Carlos Erazo-Álvarez

Capacitación: Es importante implementar la capacitación profesional en los establecimientos educativos para así lograr una mejora en el conocimiento de cada integrante con el objetivo de abordar de una manera más humana las situaciones presentadas, dar estricto cumplimiento a los seguimientos para bien de los educandos y así cumplir con todos los requerimientos expuestos en las rutas y protocolos de violencia entre pares. Entonces, para una excelencia profesional a través de la capacitación, se describe varias propuestas:

1. Cada coordinador de DECE a nivel distrital socializará las rutas y protocolos a seguir con los profesionales del DECE institucional. De igual manera, estos harán la socialización al inicio del lectivo a las autoridades y personal que labora en la institución educativa.

2. Capacitar a los docentes mediante charlas, talleres, grupos focales, eventos informativos y así lograr una unanimidad y articulación para el abordaje y cumplimiento las rutas y protocolos a seguir

3. Los integrantes del DECE deberán crear materiales informativos como infografías, trípticos, letreros para que los estudiantes se motiven a denunciar y puedan solicitar la ayuda respectiva por estos profesionales.

4. Se deberá difundir en redes sociales las rutas y protocolos de violencia entre pares.

5. Se deberá gestionar con las autoridades locales e institucionales, un programa de radio o la participación en uno, a fin de abordar temas netamente de casos de violencia (psicológica, sexual y entre pares). 
María Esther Regalado-Martínez; Darwin Gabriel García-Herrera; Sergio Constantino Ochoa-Encalada; Juan Carlos Erazo-Álvarez

\section{CONCLUSIONES}

Con fundamento en la investigacion realizada se puede concretar enunciado que existe una falta de conocimiento en relación a las rutas y protocolos de violencia entre pares por parte de la comunidad educativa de la Unidad Educativa La Providencia de la ciudad de Azogues. Aunque el personal del DECE domina los conceptos y la aplicación de las rutas y protocolos, la percepción que tiene dicho personal influye de manera directa en la aplicación de las rutas y protocolos de violencia entre pares. Asimismo, se develó que para el personal del DECE es de vital otorgarle un tinte humanistico a la formulacion de estos manuales, y por consiguiente, es indispensable la participación de psicólogos y profesionales afín a estos campos de acción.

Estos resultados son importantes comprenderlos dentro del contexto de una educación que está muy distante de constituirse solo como un servicio público, para situarse como un derecho humano universal; pues su acceso está plenamente garantizado por el Derecho Internacional de los Derechos Humanos, Pactos y Convenios Internacionales. Es así que, en Ecuador, la Constitución de la República que fue aprobada por la Asamblea Nacional Constituyente de Plenos Poderes en el 2008 y ratificada por el pueblo ecuatoriano en ese mismo año; garantiza y establece la progresividad de este derecho inherente al desarrollo humano.

Desde este plano, la educación ecuatoriana, desde sólidos fundamentos pedagógicos, se ha de desarrollar en un ambiente favorable para el aprendizaje significativo. Esta construcción del ambiente propicio requiere de sólidos fundamentos biopsicosociales y de sensores oportunos que se determinen como rutas y protocolos para la detección de conductas no apropiadas que afecten el comportamiento y la productividad del estudiante en cuanto a su rendimiento escolar; y que con el proyecto PIC se han de desarrollar en forma didáctica y pedagógica. 
EPISTEME KOINONIA

Revista Electrónica de Ciencias de la Educación, Humanidades, Artes y Bellas Artes

Año III. Vol III. N ${ }^{\circ}$ 6. Julio - Diciembre 2020

Hecho el depósito de Ley: FA2018000022

ISSN: 2665-0282

FUNDACIÓN KOINONIA (F.K)

Santa Ana de Coro, Venezuela

María Esther Regalado-Martínez; Darwin Gabriel García-Herrera; Sergio Constantino Ochoa-Encalada; Juan Carlos Erazo-Álvarez

\section{FINANCIAMIENTO}

No monetario

\section{AGRADECIMIENTOS}

A la Unidad Educativa Particular la Providencia de la cuidad de Azogues, por apoyar el desarrollo de la investigación. 
María Esther Regalado-Martínez; Darwin Gabriel García-Herrera; Sergio Constantino Ochoa-Encalada; Juan Carlos Erazo-Álvarez

\section{REFERENCIAS}

Aldana-Zavala, J. J. (2019). La competencia epistemológica en el investigador social universitario venezolano [The epistemological competence in the Venezuelan university social researcher]. Praxis, 15(1), 103-115. https://doi.org/10.21676/23897856.3091

Alfaro-Molina, I., Kenton-Paniagua, K., \& Leiva-Díaz, V. (2010). Conocimientos y Percepciones sobre violenci en los centros educayivos público [Knowledge and Perceptions about violence in public educational centers]. Enfermería Actual en Costa Rica, (18), 1-10.

Barquero-Brenes, A. (2014). Convivencia en el contexto familiar: un aprendizaje para construir cultura de paz [Coexistence in the family context: A learning to build peace culture]. Revista Electrónica "Actualidades Investigativas en Educación", 14(1),1-19.

Caballero-Grande, M. J. (2010). Convivencia escolar. Un estudio sobre buenas prácticas. Revista de Paz y Conflictos, (3); 154-169.

Chagas-Dorrey, R. (2012). La teoría de la agresividad en Donald W. Winnicott [The Aggression Theory in Donald W. Winnicott]. Perfiles educativos, 34(138), 29-37.

Duarte-Duarte, J. (2003). Ambientes de aprendizaje. Una aproximación conceptual [Learning environments. A conceptual approach]. Revista Iberoamericana De Educación, 33(1), 1-18. https://doi.org/10.35362/rie3312961

Fuertes-Grábalos, E, Plou-Lafuente, Pablo, \& Gómez-Bahillo, C. (2017). Desarrollo humano desde la perspectiva del crecimiento [Human development from the perspective of growth]. Revista de Ciencias Sociales (Ve), XXIII (4),81-97.

Gross-Martínez, M, \& Stiller-González, L. (2015). Contribución de la Técnica del Grupo Focal al Acercamiento a la Percepción Estudiantil sobre Accesibilidad en el Entorno Universitario [Technical Contribution to Focus Group Approach to Students Perception on Accessibility in University Environment]. Actualidades Investigativas en Educación, 15(1), 31-47. 
María Esther Regalado-Martínez; Darwin Gabriel García-Herrera; Sergio Constantino Ochoa-Encalada; Juan Carlos Erazo-Álvarez

Hernández, A. (2018b). Transitando por el camino de la escuela para padres, madres y representantes. Una experiencia vivida. [Walking on the way to school for parents and representatives. A lived experience]. EPISTEME KOINONIA, 1(1), 51-71. http://dx.doi.org/10.35381/e.k.v1i1.490

Hernandez, R. (2018a). Rol del psicólogo como agente primario en la violencia escolar. [Role of the psychologist as primary agent in school violence]. Educación médica, 19(3); 383. https://doi.org/10.1016/j.edumed.2017.11.002

Hernández-Romero, Y, \& Galindo-Sosa, R. (2007). El concepto de intersubjetividad en Alfred Schutz [The concept of intersubjectivity in Alfred Schutz]. Espacios Públicos, 10(20),228-240.

Martínez-Pacheco, A. (2016). La violencia. Conceptualización y elementos para su estudio [The violence. Conceptualization and elements for its study]. Política y cultura, (46), 7-31.

Mata-Diz, J, \& Penido-Martins, T. (2017). Acoso Escolar y la Protección de los Derechos de los Niños y Adolescentes [Bullying and Protection of the Rights of Children and Adolescents]. Educação \& Realidade, 42(3), 1001-1018.

Ministerio de Educación del Ecuador. (2016). Acuerdo Nro. MINEDUC-ME-2016-00046A [Agreement No. MINEDUC-ME-2016-00046-A]. Recuperado de https://n9.cl/xt2k

Ministerio de Educación del Ecuador. (2017). Protocolos de actuación frente a la violencia detectadas 0 cometidas en el Sistema Educativo. [A Action protocols against violence detected or committed in the Educational System]. Recuperado de https://n9.cl/3yyn

Neut, P. (2017). Las violencias escolares en el escenario educativo chileno. Análisis crítico del estado del arte [School violence in the chilean educational scenario. Critical analysis of the state of the art]. Calidad en la educación, (46), 222247. https://dx.doi.org/10.4067/S0718-45652017000100222 
María Esther Regalado-Martínez; Darwin Gabriel García-Herrera; Sergio Constantino Ochoa-Encalada; Juan Carlos Erazo-Álvarez

Organización de las Naciones Unidas. (2015). La Declaración Universal de Derechos Humanos. Recuperado de https://n9.cl/XE66

Papalia, D, Wendkos-Olds, S, \& Duskin-Feldman, R. (2010). Desarrollo Humano [human development].Undecima Edición. McGraw-Hill/Interamericana Editores.

Pineda-Almaraz, A., Rivera-Fong, L., Téllez, M. A., \& Ornelas, J. (2015). Percepción del bullying en alumnos y profesores del Distrito Federal [Perception of bullying in students and teachers of the Federal District]. Intercontinental de Psicología y Educación, 17(2), 121-145.

Registro Oficial 449 de 20-oct-2008. Constitución de la Republica del Ecuador [Constitution of the Republic of Ecuador]. Recuperado de https://n9.cl/hd0q

Romero, S., \& Villasmil, J. (2017). La madurez vocacional como factor determinante en el desempeño académico de los estudiantes del tercer semestre de medicina de la modalidad ADI de la Universidad Nacional Experimental Francisco de Miranda [Vocational maturity as a determining factor in the academic performance of the students of the third semester of medicine of the ADI modality of the National Experimental University Francisco de Miranda]. Revista Arbitrada Interdisciplinaria Koinonía, 2(4), 148-179.

Roque-Díaz, R. (2018). Desarrollo personal y manejo asertivo de emociones en estudiantes. [Personal development and assertive management of emotions in $\begin{array}{llll}\text { students]. } & \text { EPISTEME }\end{array}$ http://dx.doi.org/10.35381/e.k.v1i2.511

Sáez-de-Heredia, R. A. (1998). Análisis y resolución de conflictos: una perspectiva psicológica [Conflict analysis and resolution: a psychological perspective]. Bilbao : Universidad del País Vasco, Servicio Editorial = Euskal Herriko Unibertsitatea, Argitalpen Zerbitzua.

Sánchez-Carranza, R, \& Sandoval-Forero, E. (2016). El orientador de secundaria ante los conflictos y la violencia escolar [The secondary school counselor facing conflicts and school violence]. Ra Ximhai, 12(3),47-55. 
EPISTEME KOINONIA

Revista Electrónica de Ciencias de la Educación, Humanidades, Artes y Bellas Artes

Año III. Vol III. N ${ }^{\circ}$ 6. Julio - Diciembre 2020

Hecho el depósito de Ley: FA2018000022

ISSN: 2665-0282

FUNDACIÓN KOINONIA (F.K).

Santa Ana de Coro, Venezuela

María Esther Regalado-Martínez; Darwin Gabriel García-Herrera; Sergio Constantino Ochoa-Encalada; Juan Carlos Erazo-Álvarez

Vera-Mora , G., \& León-Acurio, J. V. (2017). Cyberbullying: Una realidad de intimidación en las Unidades Educativas del Ecuador [Cyberbullying: A reality of intimidation in the Educational Units of Ecuador]. Revista Atlante. Cuadernos de Educación y Desarrollo. [Internet]. Recuperado de https://n9.cl/7l7g

C2020 por el autor. Este artículo es de acceso abierto y distribuido según los términos y condiciones de la licencia CreativeCommons Atribución-NoComercial-Compartirlgual 4.0 Internacional (CC BY-NC-SA 4.0) (https://creativecommons.org/licenses/by-nc-sa/4.0/). 